\title{
Relevance of workplace social mixing during influenza pandemics: an experimental modelling study of workplace cultures
}

\author{
T. TIMPKA ${ }^{1,2,3 *}$, H. ERIKSSON ${ }^{2}$, E. HOLM ${ }^{4}$, M. STRÖMGREN ${ }^{4}, J_{\text {J EKBERG }}^{1,3}$, \\ A. SPRECO ${ }^{1,2}$ AND Ö. DAHLSTRÖM ${ }^{5}$ \\ ${ }^{1}$ Department of Medical and Health Sciences, Linköping University, Linköping, Sweden \\ ${ }^{2}$ Department of Computer and Information Science, Linköping University, Linköping, Sweden \\ ${ }^{3}$ Unit for Health Analyses, Östergötland Healthcare Region, Linköping, Sweden \\ ${ }_{5}^{4}$ Department of Geography and Economic History, Umeå University, Umeå, Sweden \\ ${ }^{5}$ Department of Behavioural Sciences and Learning, Linköping University, Linköping, Sweden
}

Received 15 July 2015; Final revision 10 January 2016; Accepted 14 January 2016; first published online 5 February 2016

\section{SUMMARY}

Workplaces are one of the most important regular meeting places in society. The aim of this study was to use simulation experiments to examine the impact of different workplace cultures on influenza dissemination during pandemics. The impact is investigated by experiments with defined socialmixing patterns at workplaces using semi-virtual models based on authentic sociodemographic and geographical data from a North European community (population 136000). A simulated pandemic outbreak was found to affect $33 \%$ of the total population in the community with the reference academic-creative workplace culture; virus transmission at the workplace accounted for $10.6 \%$ of the cases. A model with a prevailing industrial-administrative workplace culture generated $11 \%$ lower incidence than the reference model, while the model with a self-employed workplace culture (also corresponding to a hypothetical scenario with all workplaces closed) produced $20 \%$ fewer cases. The model representing an academic-creative workplace culture with restricted workplace interaction generated $12 \%$ lower cumulative incidence compared to the reference model. The results display important theoretical associations between workplace social-mixing cultures and community-level incidence rates during influenza pandemics. Social interaction patterns at workplaces should be taken into consideration when analysing virus transmission patterns during influenza pandemics.

Key words: Epidemiology, infectious disease control, influenza, medical informatics (veterinary and medical), modelling.

\section{INTRODUCTION}

There has to be at least two meeting places with partly overlapping and volatile regular visitors in a community in order to start an outbreak of any airborne

\footnotetext{
* Author for correspondence: Professor T. Timpka, Division of Social Medicine and Public Health, Department of Medical and Health Sciences, Faculty of Health Sciences, Linköping University, SE-581 83 Linköping, Sweden.

(Email: toomas.timpka@liu.se)
}

infectious disease pathogen [1, 2]. Although workplaces are one of the most important locations for social mixing in society, few studies have addressed the role of workplace virus transmissions during influenza pandemics [3]. On an average business day, one third of the Swedish adult population dwells at a workplace for $>7 \mathrm{~h} \mathrm{[4]}$ and during that work time, some $60 \%$ have physical contact with at least one of the other 20 persons (median) present in their section of the workplace. However, the social-mixing cultures at 
workplaces may differ with both the internal organization of the enterprise and norms in the surrounding society [5-9]. These facts justify efforts to investigate the role of workplaces in influenza transmission during pandemics and the influence of different workplace cultures for meetings and social mixing.

The aim of this study was to use controlled simulation experiments where elements external to the research question are kept constant to examine the impact of social mixing at workplaces on influenza dissemination during pandemics. The specific research question to be answered was what impact the social organization of work (workplace culture) has on municipality-level influenza incidence rates. The study is part of a research programme with the aim of using time-geographical theory, empirical data, and computer model experiments to investigate the expected impact from social distancing and behavioural interventions in different areas of society during pandemic influenza outbreaks $[10,11]$. The experiments were performed in a simulation environment containing authentic sociodemographic and geographical data from a North European municipality (population 136000). The simulation model was constructed using an ontology system [12, 13].

\section{Empirically based social pockets as a basis for modelling influenza outbreaks}

A key element (as exogenously given or endogenously calculated) in any model of influenza outbreaks is the distribution over all individuals of the probability that person $p$ transmits the influenza virus to person $q$ for given time period during an outbreak. Two main components determine this transmission probability; the social component is the probability that $q$ meets $p$ and the probability that the physical interaction during the meeting results in $q$ being exposed to the virus; the biological component is defined dynamically by the biological status (susceptible, infectious, recovered, etc.) of $p$ and $q$, respectively. For the social component, most models use differentiating and grouping of the population in order to replace the globally equal meeting probability with specific estimates closer to the level relevant for different persons in different situations. Mainly two types of methodologies are used: (1) differentiating individual probabilities by attributes such as age [14, 15] or social status [16]; and (2) grouping of individuals into social networks or associating them with spatial meeting places. The representation of social network groupings [17-19] has recently been extended to include also dynamic changes in the network during outbreaks [20]. Groupings of individuals based on spatially defined meeting places have used locations such as homes, workplaces, schools, and neighbourhoods with a selected limited set of joint co-visitors [21, 22]. The rationale behind such spatial groupings of individuals is that if the members and their length of stay in the same concrete instance of a spatially defined meeting place can be identified, the meeting and transmission probabilities can be set higher for persons within the groups compared to between groups. That, in turn, combined with differentiation by attributes, enforces a better distinction of who transmits to whom on a particular day. The challenge then is to define and find a reasonable set of spatially defined meeting places including an empirically based mechanism outlining who will enter and leave these meeting places and for how long they stay and how closely they interact physically.

A theoretically founded approach to inform spatially explicit simulations of infectious disease outbreaks is to depart from Hägerstrand's concept of pockets of local order [23]. One advantage of conceptualizing such pockets in infectious disease modelling is that emerging behaviour and contact patterns within them can be modelled beyond the aggregate of their members' attributes. The distribution of contact probabilities between the members can be modified by size and the specific culture in a specific pocket [24]. A structure based on social relationships, transportation routines, and other movement patterns is then used to represent the mixing groups in communities as nodes with contact frequencies between individuals within them as well as the other properties of social pockets. In other words, a fixed arbitrary hierarchy of household groups and regions is replaced by a system of meeting-place centred, floating, and overlapping reference areas for social mixing with different location, meeting and member properties [25].

There is sparse empirical data available to directly represent different types of social interactions at workplaces in models of influenza transmission. Instead, modelling experiments can be used to explore the effects of theoretically postulated scenarios on transmission probabilities. Influenza transmission patterns at workplaces can be modelled based on empirical data on workplace social mixing cultures and then use simulations to calculate the wider consequences of those workplace transmission models on total societal incidence rates. In order to narrow down the 
possible influences on transmission rates to be expected by certain workplace social distance settings, stylized example workplace social interaction cultures can here be outlined for use in model experiments.

\section{METHODS}

The study design was based on simulation experiments. The impact of different workplace cultures on local influenza incidence during pandemics was investigated by modelling different workplace cultures in a simulation environment containing authentic sociodemographic and geographical data from a North European municipality by combining workgroup sizes and withinworkgroup person-to-person transmission probabilities while keeping other mixing-group properties constant. A community-based model of influenza transmission was configured for municipality scenarios where the social mixing at workplaces was assumed to follow academic-creative (reference model), industrial-administrative, and self-employed cultures, respectively. Furthermore, the experiment included an additional social distancing scenario based on the academic-creative workplace culture, but where the close person-to-person contacts associated with risk for influenza transmission were reduced to half.

\section{Ethics statement}

The present research programme is based on administrative public health databases established for the purpose of systematically and continuously developing and securing the quality of health service. For this study based on mathematical modelling and simulation, only demographic data from Statistics Sweden (http://www.scb.se/en_/) were used. No authentic health data were included in the analyses. Because the study design only employed simulated infectious disease data it was given exemption by the regional ethics committee.

\section{Representation of workplace social mixing}

In the industrial-administrative workplace culture, workers meet closely with other workers mainly on a one-to-one basis. For example, they might be assigned to a machine or desk with space for only one additional person close by. This situation corresponds to a constant one-to-one total transmission probability for each infected worker, independent of the number of other workers around. In previous infectious disease modelling experiments, this culture has been represented by creating explicit workplace mixing groups in the model and setting the number of interacting workers in each workplace mixing group to the mean number of surrounding co-workers suggested by empirical data $(n=20)[8,26,27]$. Correspondingly, this workplace social-mixing model was used in the simulation experiments to represent to the industrial-academic workplace culture. In comparison, when adhering to an academic-creative workplace culture, workers are located in a common indoor space and a substantial part of their working time is devoted to physical meetings with subsets of other workplace members. This situation corresponds to a constant probability that an infected worker may transmit the virus to any other worker in the workgroup. Since the exposure is simultaneous for all workgroup members, the theoretical transmission probability is multiplicatively related to the total exposure time and to the number of workgroup members. However, Swedish empirical data [4] show that when the size of the workgroup increases from 1 to $>50$, the average number of physical contacts per person increases from around 1 to 2 (Fig. 1). In the simulation experiments, this scenario was therefore represented by having explicit workplace-mixing groups in the model and doubling the number of workers in each workplace-mixing group (to $n=40$ ) while keeping the person-to-person transmission probability constant. Finally, in the self-employed workplace culture, represented, for instance, by farmers and small-scale business owners in rural areas, workers do not meet other workers during the working day. This situation was represented in the simulation experiments by not having any workplaces at all in the model. Most workers are assumed to spend most of their working time interacting with artifacts, tools, and equipment rather than people.

\section{Simulation dynamics}

The model of influenza transmission consists of a spatially explicit community component, which is based on mixing groups representing locations where individuals meet [26, 28], and a biological component representing infectious disease properties, such as the duration of the latent and incubation periods. The details of the societal and biological components are provided in the online Supplementary material. Typically, each person belongs to several mixing groups where infectious participants may transfer the influenza virus to them [29]. 


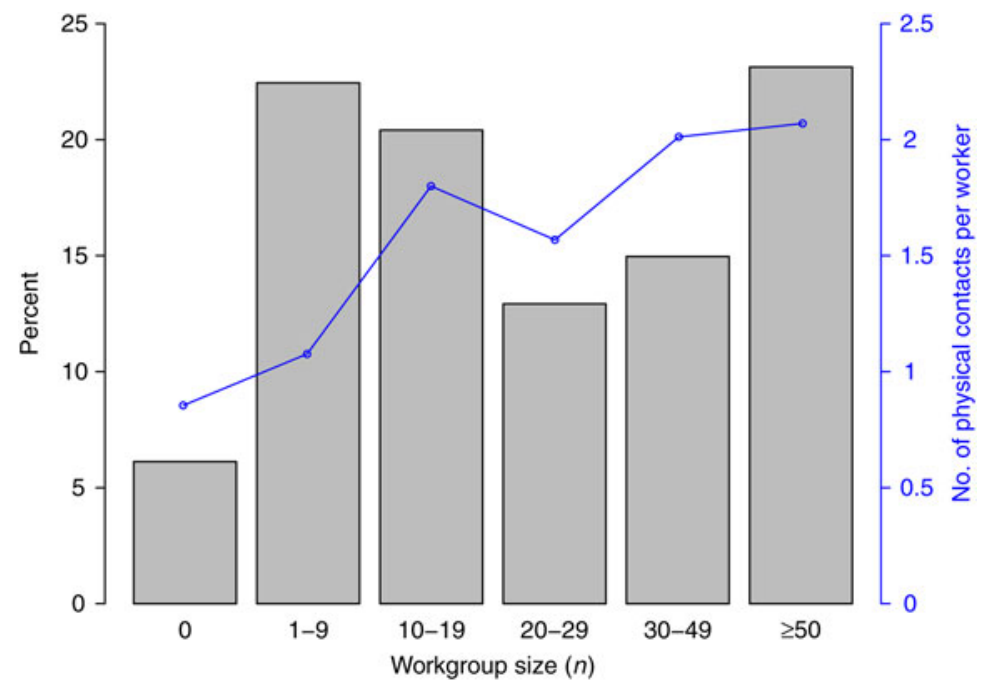

Fig. 1. Swedish workforce displayed by the proportion of workgroup sizes and the number of other workers that a worker has been in physical contact with during 1 day (adapted from [4]). Workgroup size $n=0$ denotes that the worker is self-employed and does not belong to a workgroup.

Before the simulation starts, the individuals are assigned to their mixing groups based on population data. To adjust for situations not covered by authentic population data, such as cohabiting young adults (unmarried without children), the simulator randomly merges a specified set of the single households to form two-adult households [30].

At the start of a simulation experiment, the model is seeded by randomly infecting a specific number of individuals. For the present study, the simulator seeded $1 / 1000$ individuals $(n=136)$ as index cases to represent a standardized scenario with a sufficient number of cases to initiate a local outbreak of pandemic influenza. Using the index cases, the simulator calculates stochastically generated secondary infections based on the transmission probabilities in the model. The main loop steps through a specific number of time periods (24-h days) covering the expected duration of the outbreak. The simulator runs iterations for each day, updating the infectious status of persons and withdrawing them from their mixing groups to home when they are symptomatic. For each day, the simulator iterates through all susceptible persons and calculates the probability of infection based on the mixing groups they participate in using the complementary event of infection; that is, the multiplied probability of escaping infection from each of the infected people that the individual meets in the mixing groups he/she participates in during the day $[12,13,31]$. Symptomatic individuals withdraw from their mixing groups to the household with an age-adjusted probability.
The value for the probability of infection is then compared with a generated random number to decide whether to infect a person or not in the simulation run. If the result is to activate the infection for an individual on the current day, the simulator uses the specific biological model to generate a secondary case and set the initial state of the infection to latent. This model guides the generation of the case, including the duration of the latent, incubation, and infectious periods. During the simulation process, the biological model updates the infectious status for each individual for each day, for example, by changing the status of viral shedding. The simulator also keeps track of individuals' infectious disease status in each of the mixing groups and uses this information to calculate the probability of infection in the next iteration (i.e. the next day in the simulation).

\section{Assignment to workplaces and workgroups}

The representation of mixing groups in the model uses counterparts to residents and meeting places in Linköping municipality, Sweden (Table 1 and Supplementary Table S2). The apparatus for creating a realistic heterogeneity in transmission probabilities is based on a set of socio-culturally well-defined meetingplace types with a spatial duplication of separate mixing-group instances of the same type (Supplementary Tables S3 and S4). In addition, this model utilizes authentic locations of homes, workplaces, and schools instead of fictitious locations. In the model, the members in the 
Table 1. Study population displayed by age and gender

\begin{tabular}{lrrr}
\hline \hline Age (years) & \multicolumn{1}{l}{ Male } & \multicolumn{1}{l}{ Female } & \multicolumn{1}{l}{ Total } \\
\hline $0-9$ & $7494(10 \cdot 9)$ & $7114(10 \cdot 5)$ & $14608(10 \cdot 7)$ \\
$10-19$ & $8895(13 \cdot 0)$ & $8267(12 \cdot 2)$ & $17162(12 \cdot 6)$ \\
$20-29$ & $12248(17 \cdot 9)$ & $10698(15 \cdot 8)$ & $22946(16 \cdot 8)$ \\
$30-39$ & $10260(15 \cdot 0)$ & $9147(13 \cdot 5)$ & $19407(14 \cdot 2)$ \\
$40-49$ & $8664(12 \cdot 7)$ & $8137(12 \cdot 0)$ & $16801(12 \cdot 3)$ \\
$50-59$ & $8336(12 \cdot 2)$ & $8380(12 \cdot 4)$ & $16716(12 \cdot 3)$ \\
$60-69$ & $5918(8 \cdot 6)$ & $6352(9 \cdot 4)$ & $12270(9 \cdot 0)$ \\
$70-79$ & $4188(6 \cdot 1)$ & $5301(7 \cdot 8)$ & $9489(7 \cdot 0)$ \\
$80-89$ & $2195(3 \cdot 2)$ & $3719(5 \cdot 5)$ & $5914(4 \cdot 3)$ \\
$\geqslant 90$ & $247(0 \cdot 4)$ & $671(1 \cdot 0)$ & $918(0 \cdot 7)$ \\
Total & $68445(100)$ & $67786(100)$ & $136231(100)$ \\
\hline \hline
\end{tabular}

Values given are $n(\%)$.

Source: Statistics Sweden (http://www.scb.se/en_/)

different mixing-group episodes are constant for the duration of the simulation. Therefore, system-wide diffusion comes about by people being members of more than one mixing group, receiving the pathogen in one group and transmitting it on another day in another group.

For employed individuals (aged 18-65 years), the simulator uses a workplace model consisting of mixing groups based on a combination of workplace data and assumed internal workplace structures (workgroups). Since the employment data relate individual employees to formal workplaces and organizational identifiers (rather than actual workgroups), the model divides the workforce into separate workgroups with their own mixing patterns (Supplementary Table S5). This change in workgroup structures is done so that different workplace cultures can be compared. Furthermore, the model assumes that $5 \%$ of the workforce is absent at any given time, for instance due to other types of illness and parental leave. The simulator selects absentees randomly before the simulation starts. Moreover, the simulator uses a weekly schedule where workplaces and schools are closed during weekends (i.e. the simulator disables all transmission in the workplace, daycare, and school mixing groups).

\section{Statistical analyses}

The impact of workplace cultures on the local development of pandemic influenza was analysed using modelling experiments covering each of the following four conditions: (a) an academic-creative workplace culture prevailed throughout the community, (b) an academiccreative workplace culture prevailed and a social distancing intervention was in place at workplaces decreasing workplace virus transmission probability by $50 \%,(c)$ an industrial-administrative workplace culture prevailed, and $(d)$ a self-employed workplace culture prevailed throughout the community. Data from 100 outbreaks in the same semi-synthetic population were collected for each condition and outbreak durations and influenza case rates were estimated by calculating means, prediction intervals (PIs; computationally similar to confidence intervals for sampling errors in distribution of observables), and ranges based on 100 simulation runs (range ${ }^{100}$ ). In these analyses, there was no traditional sampling error because each individual in the population was contained in the analyses. The entities investigated are not observables but the outcomes of purposefully generated stochastic processes with systematic components. Repeating such simulations makes two pieces of information available: (1) an estimate of the mean outcome, which is more reliable than the result of a single simulation as an approximation of the combined effect of the systematic influence from the simulation apparatus and the influence of the controlled conditions, and (2) a measure of the distribution of outcomes around the mean outcome, in this study represented by the range ${ }^{100}$. To avoid making further type 2 inference errors when examining the results of repeated simulations, the $95 \%$ PI (mean of 100 simulations \pm 1.96 s.D.) for single model replications were calculated for an index based on the reference model. IBM SPSS Statistics for Windows, v. 21 (IBM Corp., USA) was used for the analyses.

\section{Sensitivity analyses}

To validate the soundness of the parameter estimates kept constant during the simulation experiments, six series of sensitivity analyses, each comprising 100 simulation runs, were computed. The community with an industrial-administrative workplace culture was used as baseline. In the first two series, the person-to-person influenza transmission probability at high schools was reduced to half and increased twofold, respectively. In two additional series, the probabilities that symptomatically ill individuals withdraw to their household for age groups $0-5,6-18$, and $\geqslant 19$ years $(0 \cdot 80,0.75$ and $0 \cdot 50)$ were changed to very low presence in activities outside the home $(0.95,0.90$ and 0.80$)$, and high presence $(0 \cdot 70,0 \cdot 50$, and $0 \cdot 30)$. Finally, to understand the impact from random mixing in the community on influenza transmission, the probability for community transmission of influenza virus was decreased to half 
Table 2. Duration in days of standardized outbreaks with 136 initially infected individuals in a population of 136000. Means and ranges of 100 simulation runs (range $e^{100}$ ) are presented together with a duration index (index) based on proportions compared with the reference model. The $95 \%$ PI (mean of 100 simulations \pm 1.96 S.D.) for single model replications are added for the duration index

\begin{tabular}{lllclc}
\hline \hline Workplace culture & Duration (days) & Range $^{100}$ (days) & Index & Range $^{100}$ (index) & $95 \%$ PI (index) \\
\hline Academic-creative (reference) & 92 & $86-100$ & 100 & $93-108$ & $95-105$ \\
Industrial-administrative & 87 & $81-98$ & 95 & $88-106$ & $88-101$ \\
Academic-creative restricted & 87 & $82-92$ & 94 & $89-100$ & $89-99$ \\
Self-employed & 87 & $80-96$ & 94 & $87-104$ & $87-101$ \\
\hline \hline
\end{tabular}

PI, Prediction interval.

Table 3. Cumulative incidence of those infected with pandemic influenza in a population of 136000 . Means and ranges of 100 simulation runs (range ${ }^{100}$ ) are presented together with an incidence index based on proportions compared with the reference model. The $95 \%$ PI (mean of 100 simulations \pm 1.96 S.D.) for single model replications are added for the incidence index

\begin{tabular}{llllll}
\hline \hline Workplace culture & $\begin{array}{l}\text { Cumulative } \\
\text { incidence }(n)\end{array}$ & $\operatorname{Range}^{100}(n)$ & Index & $\begin{array}{l}\text { Range }^{100} \\
\text { (index) }\end{array}$ & $95 \%$ PI (index) \\
\hline $\begin{array}{l}\text { Academic-creative } \\
\text { (reference) }\end{array}$ & 45037 & $43834-46132$ & 100 & $97-102$ & $98-102$ \\
$\begin{array}{l}\text { Industrial-administrative } \\
\text { Academic-creative restricted }\end{array}$ & 40103 & $39209-41282$ & 89 & $87-92$ & $87-92$ \\
Self-employed & 39810 & $38524-41490$ & 88 & $86-92$ & $86-91$ \\
\hline \hline
\end{tabular}

PI, Prediction interval.

in one series of 100 simulation runs and increased threefold in another.

\section{RESULTS}

The mean duration of the reference outbreak was 92 days (range ${ }^{100} 86-100$ days) (Table 2). The mean duration of the outbreaks generated in the other models did not differ from the reference model. The reference academic-creative workplace model produced a mean cumulative incidence of 45037 infected individuals (33\% of the 136231 individuals in the total population) (Table 3). The industrial-administrative workplace culture model generated an $11 \%$ lower mean cumulative incidence (40 103, range ${ }^{100} 39209$ - 41 282) than the reference scenario ( 45037 , range ${ }^{100} 43$ 834- 46132 ) and the $95 \%$ PI of the single replication index for the same model (index 89\%, 95\% PI $87-$ 92) did not overlap with the corresponding $95 \%$ PI of the reference model (index 100\%, 95\% PI 98102). The $11 \%$ difference can therefore be regarded as statistically noteworthy with regard to repeated replications of simulations and larger than what is required for statistical significance related to one single replication. The same inferences can be made regarding the differences in cumulative incidence between all other models, except for the models representing the industrial-administrative (index 88\%, 95\% PI 87-92) and the restricted academic-creative (index $89 \%$, 95\% PI 86-91) workplace cultures. The model representing an academic-creative workplace culture with restricted workplace interaction generated a $12 \%$ lower cumulative influenza incidence (index $88 \%$, 95\% PI 86-92) than the reference model, while the model with a self-employed workplace culture produced a 20\% lower incidence (index 80\%, 95\% PI 79-82).

\section{Location of virus transmission}

The household was the meeting place accounting for the largest share of influenza virus transmission in all experimental conditions; from $29 \%$ of the transmissions in the academic-creative workplace culture model to $33 \%$ of the virus transmissions in the selfemployed workplace culture model (Fig. 2). However, while the proportion infected at workplaces was $10 \cdot 7 \%$ under academic-creative workplace culture conditions, this proportion was about half as large 


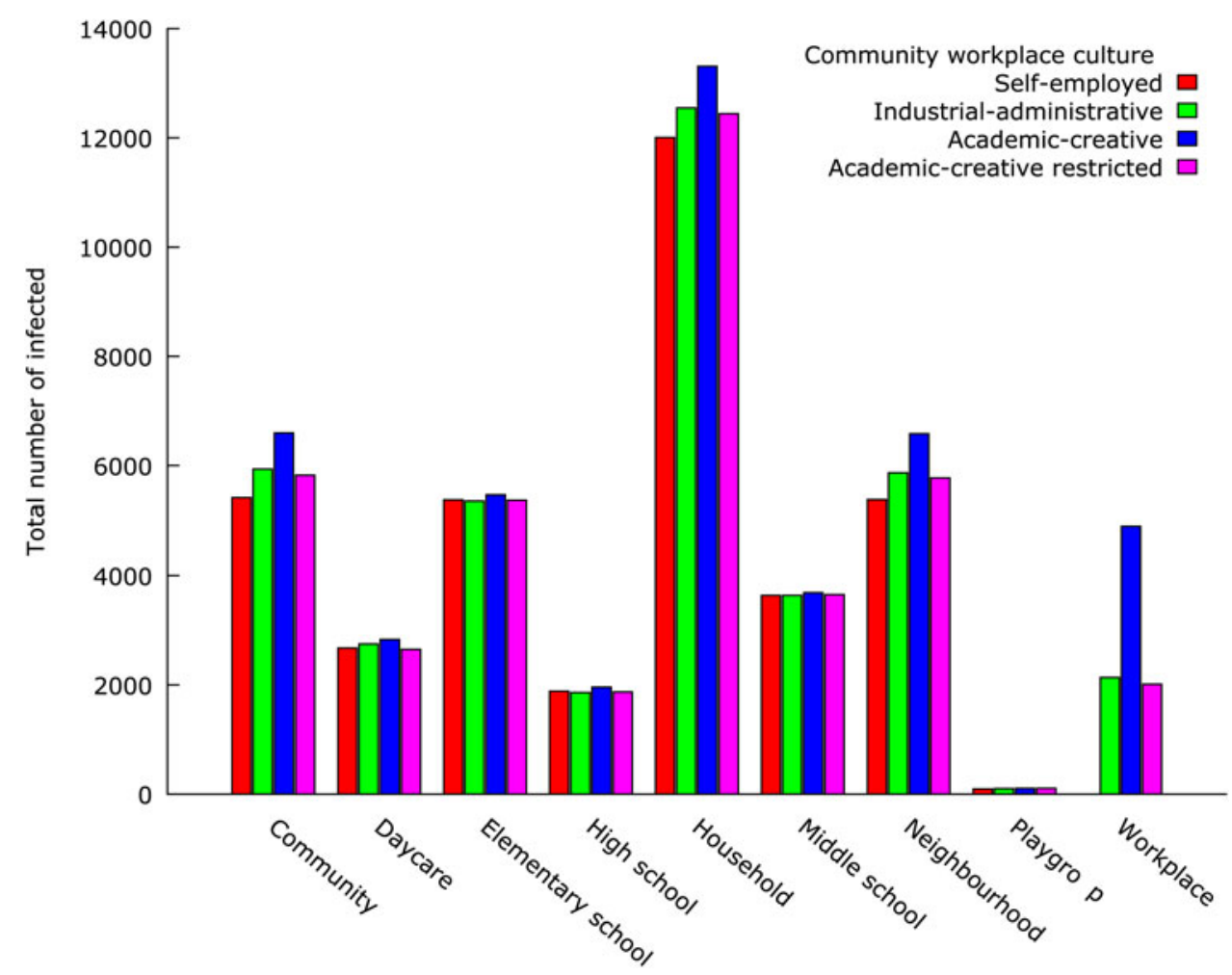

Fig. 2. Locations for influenza transmission in the semi-virtual community (population 136 231) during a pandemic outbreak displayed by the workplace culture model.

$(5 \cdot 3 \%)$ under industrial-administrative workplace culture conditions.

When investigating the two academic-creative workplace models more closely, it was found that the main location for virus transmission in the reference model was the household $(29 \%, n=13275)$ (Fig. 3). Next in importance were neighbourhoods $(14 \%, n=$ 6513), community-level mixing (14\%, $n=6506)$, elementary schools $(12 \%, n=5435)$, and workplaces $(11 \%, n=4812)$. Somewhat fewer cases originated from transmission at middle schools $(8 \%, n=3670)$, daycare centres $(6 \%, n=2825)$, and high schools $(4 \%, n=1901)$. Comparing these numbers with the numbers generated in the restricted academic-creative workplace model, the main effect on influenza transmission was found to remain local to the workplaces. Out of the 4812 workplace virus transmissions observed in the reference model, $58 \%(n=2792)$ disappeared as an effect of the $50 \%$ reduction in transmission probability. Closer analysis of the reduction gained at workplaces and the total virus transmission in all mixing groups shows that the presumed social distancing intervention leading to a $50 \%$ reduction of risk for influenza transmission at workplaces multiplied into a total system-wide reduction of 5226 cases (a factor of 1.9). The indirect reduction in the other mixing groups visited by (now, fewer) contagious workers was not distributed between the mixing groups in proportion to the total number of cases in the mixing groups without the intervention. This is because the joint distribution of memberships in several mixing groups is not random. Besides the internal effect in workplaces ( $53 \%$ of the total reduction), the main indirect reduction occurred in households $(15 \%)$, at the community level $(13 \%)$, and in neighbourhoods $(14 \%)$ (Fig. 4). The effects in the remaining mixing groups (schools and daycare) were minor, revealing that, as this model specified, only a few workers also visit places where young people spend their day time.

\section{Sensitivity analyses}

Regarding the influence from parameter settings for influenza transmission at high schools, the mean cumulative incidence in the industrial-administrative community decreased by $6 \%$ from 40103 (range $^{100}$ $39209-41282$ ) to 37859 (range $^{100} 36820-39276$ ) when person-to-person transmission probability was reduced to half. By contrast, when the transmission probability was increased twofold, the cumulative 


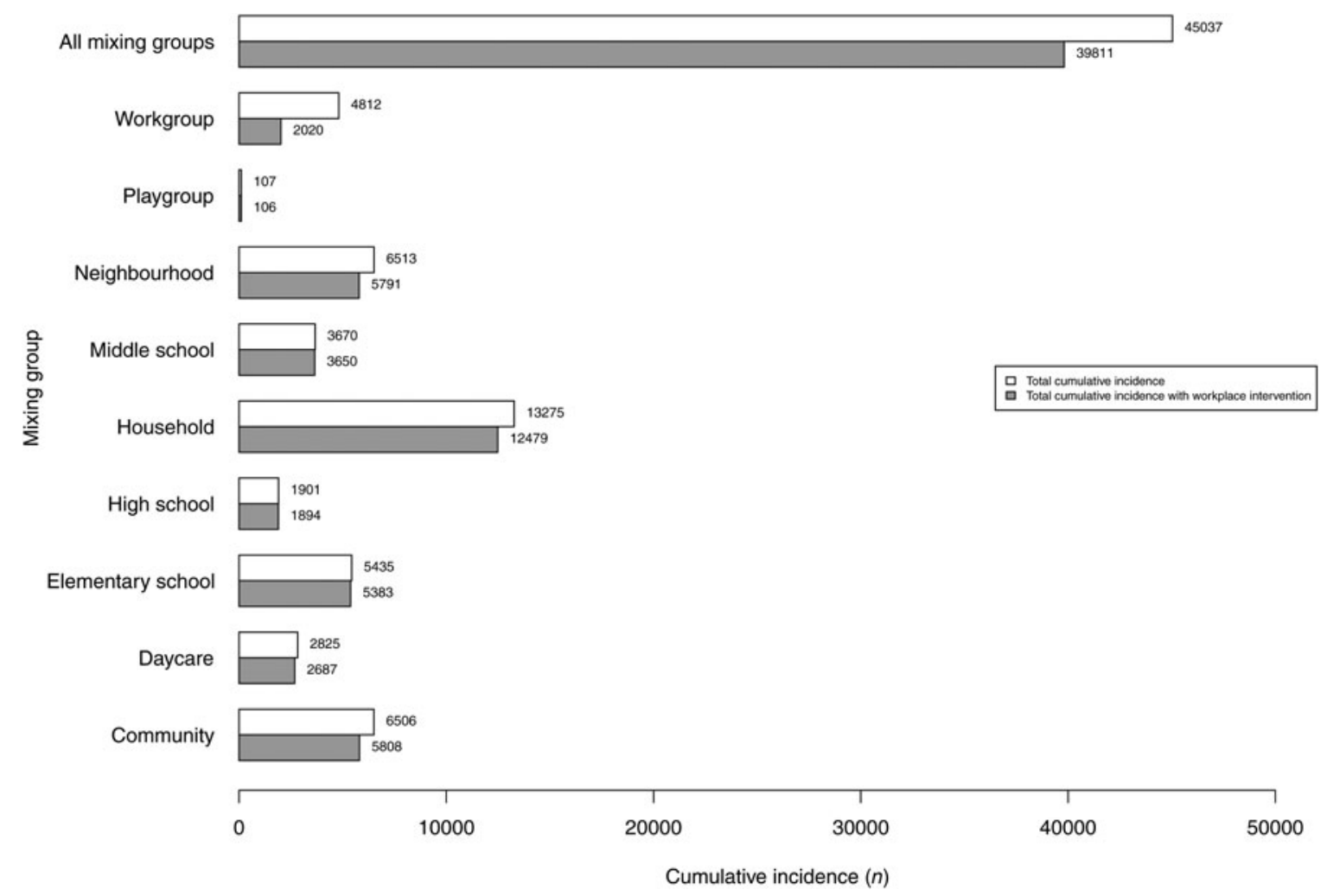

Fig. 3. Locations for influenza transmission in the academic-creative workplace culture model displayed by mixing group. The white bars show the cumulative incidence of individuals infected in the different locations in the baseline setting. The grey bars demonstrate the corresponding cumulative incidence when the risk for influenza transmission at workplaces is decreased by $50 \%$.

incidence increased by $6 \%$ to 42376 (range $e^{100} 41135-$ 43628 ) infected individuals. When the probabilities for symptomatically ill individuals withdrawing from activities outside their household were increased to a level at the high extreme, the cumulative incidence in the community decreased by $36 \%$ to 25666 (range ${ }^{100} 24$ 107-27 052) infected individuals. In comparison, when the probabilities for withdrawal were decreased to a level at the low extreme, the mean cumulative incidence increased by $51 \%$ to 60496 (range $^{100} 59$ 230-61 343). Finally, when the probability for community transmission of influenza virus was reduced to half, the mean cumulative incidence decreased by $16 \%$ to 33868 (range ${ }^{100} 32808-35$ 370). Correspondingly, when this probability for community transmission was increased threefold, the mean cumulative incidence increased by $87 \%$ to 74 867 (range ${ }^{100} 73$ 165-75 999) infected individuals.

\section{DISCUSSION}

The aim of this study was to examine the impact of workplace social mixing on community-level influenza transmission during pandemics. Using simulation experiments, we found that a reference community with an academic-creative workplace culture generated a $12 \%$ higher cumulative incidence of infected individuals than a community with an industrial-administrative workplace culture, while a community with a self-employed culture showed a $20 \%$ lower cumulative incidence than the reference community. Moreover, a hypothesized 50\% reduction in transmission probability in the workplace setting led, through system effects, to a $58 \%$ decrease in influenza transmission at workplaces. Comparing the reduction of transmissions gained at workplaces with the total reduction in the community (Fig. 4), an additional secondary reduction effect, as large as the primary effect, was observed based on the fact that individuals belonging to several different mixing groups escaped infection and thus did not transmit the virus.

These results demonstrate the importance of including detailed representations of social mixing at workplaces in community-level infectious disease modelling. Although workplaces are regularly included in modelling of influenza pandemics [32, 33] and 


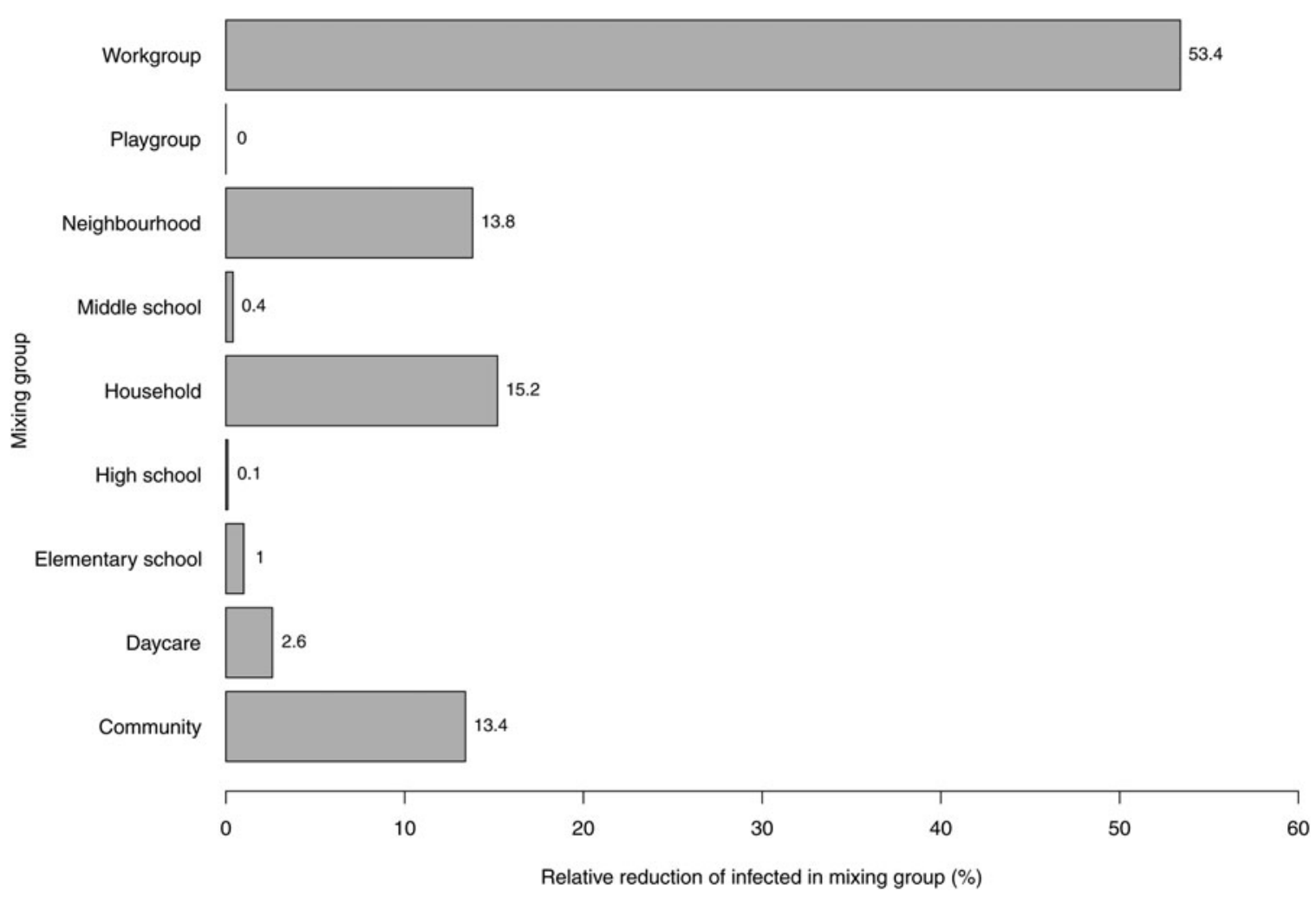

Fig. 4. Distribution of mixing groups in the percentage total reduction in influenza transmission resulting from reducing the transmission probability at workplaces by $50 \%$ (with reference to the academic-creative workplace culture).

factors such as workplace presenteeism have also been studied in some detail [34], the impact of differences in social interaction cultures at workplaces has not been studied. Our results also highlight the importance of the core behavioural assumption to what extent the contact intensity between pairs of subjects is related to the number of persons present at the type of meeting place. In this study, the extreme alternatives of this assumption in the workplace context are mirrored in the operationalization of the academic-creative and selfemployed workplace cultures. Representations of these cultures in workplace-mixing groups produced significantly different results with regard to influenza incidence at the community level, which implies that it is essential to get as much empirical support as possible for assumptions used in modelling of workplace mixing in settings where references are made to realworld communities. There are several empirical studies available on contact rates between individuals in normal populations [35-37] but few studies focusing on interaction at workplaces. However, one recent investigation [4] reported that when the subjective workgroup size increases from 1 to $>50$, the average number of physical contacts per person only increases from around 1 to 2 . That result is far from the proportional increase from 1 to 50 that would correspond to the assumption of a constant transmission probability among all (susceptible respective infectious) mixing group members. More research in this area is therefore warranted.

The four model experiments performed to answer the research questions in this study reflect different assumptions about workplace culture at the local level and its effect on the development of influenza pandemics. However, it is also possible to interpret comparisons between some of the experiments as intervention effects. Comparing the outcome of the experiment with an academic-creative workplace culture with the restricted version of the same alternative is one example of viewing the difference as an intervention outcome. The model settings for the two experiments were identical except that the interpersonal transmission probabilities at workplaces were reduced by $50 \%$ in the restricted scenario. Therefore, the difference in outcome between the two experiments mirrors the effect of implementing social distancing and hygiene measures that reduce the workers' exposure to the airborne pathogen at the workplace by half. Thus, if large parts of the working population spend time in an academic-creative workplace culture, 
employers could be asked to temporarily ban seminars and group meetings. If this intervention approach turns out to be insufficient, the duration, closeness, and frequency of one-to-one meetings can be restricted and physical meetings replaced to a large extent with e-mail, telephone, and electronic conferences, in effect transforming workplaces into a self-employed workplace culture. Moreover, the results of the experimental analyses of workplace social mixing revealed that introducing workplaces with an academic-creative workplace culture into the community model generates a $20 \%$ higher cumulative influenza incidence than using a community model without any workplaces. A further interpretation of this observation is that significant community-level transmission would remain when closing all workplaces during an entire pandemic outbreak. In addition, the results show that closing workplaces would only marginally influence the time to peak and the duration of the outbreak. These observations not only imply that economically costly interventions at workplaces should be focused on high-risk settings but also that social distancing interventions at workplaces can be efficient even relatively late during an outbreak.

This study has some strengths and weaknesses that should be taken into consideration when interpreting the results. The advantage of model experiments on virus diffusion during outbreaks of pandemic influenza in a semi-virtual model of the individuals and meeting places in a factual community is that a computer-based simulation enables grand-scale artificial experiments with changed conditions that would never be practically, ethically, or economically feasible to prepare directly in the factual population $[33,38]$. The inference from the simulation outcomes in the artificially created world of agents and mixing places into the world outside the model is entirely contingent on the accuracy of the model representation of the conditions for and the behaviour of people and viruses [11]. The inferences made in this study are contingent on the assumptions underpinning the semi-virtual model. In other words, the results regarding changes in cumulative influenza incidence are valid primarily with regard to the constructed model, and external generalizations have to be made with care. Nevertheless, the study results imply that the prevalence of different workplace cultures in the community should be considered when planning to implement social distancing interventions.

Moreover, the methods and standards for drawing inferences from analyses based on semi-virtual simulation models have not been completely established. It should be remembered that what is theoretically regarded to occur outside the simulation model in the target community corresponds to just one and only one replication, and never to an average of 100 developments. Therefore, the distribution of single outcomes around the mean (range ${ }^{100}$ ) is the only distribution that would also retrospectively eventually contain one replication close to the one that will actually emerge. In order to bridge the gap to the traditional interpretation, one can envision 1 or 100 process replications as a sample drawn from the super population of all possible developments that could be produced by the simulation model as specified (and, if the model is correctly specified, from the super population of all possible influenza developments that could occur in the target community outside the model). The distribution of outcomes in that sample mirrors the joint effect of all pure stochastic choices in the model (i.e. which specific susceptible person of a certain sex and age in a certain mixing group will become infected at a specific point in time), choices that deliberately (or rather by necessity due to lack of knowledge) were not represented in the current analyses. Instead, within the constraints given by the systematic model content, such specific events were implemented as random choices and so produced slightly different aggregate outcomes in each model replication. However, contrary to the case with a sample from given observables, this random error is not the only error or even the most important error in the outcome of a simulation. Instead, the most important errors are specification errors in the causal system setup, in assumptions, and in parameter estimates. Those errors are hidden within the mean outcome. The evident option for pinpointing such errors is to perform series of simulations with different settings for the parameter values kept constant during the simulation experiments. In the sensitivity analyses performed in this study, the alteration of parameter values for high-school transmission rates led to effects on community incidences in the same moderate range as observed in previous studies [39]. As also previously reported [34, 40], changing withdrawal probabilities for symptomatic individuals to extreme values led to notable effects on the community-level cumulative incidence of influenza cases. Empirical data on presenteeism of symptomatic individuals at workplaces during pandemic outbreaks are scarce, but the information available suggest that withdrawal is contingent both on the virulence of the circulating influenza 
strain and factors associated with sickness compensation [41, 42]. Evidently, more empirical research on workplace presenteeism during influenza pandemics is warranted. Likewise, increasing the probability for virus transmission by random mixing in the community augmented rather markedly, as expected, the community-level cumulative incidence. Reliable empirical data on the most important locations for influenza virus transmission during pandemics are scare. Reviews have suggested that the main locations for transmission of influenza virus during outbreaks vary among countries and settings, with differences in estimates also being due to differences in study designs $[43,44]$. These observations imply that more empirical research is warranted also on the spatial distribution of virus transmission during influenza pandemics. Summarizing the results from the sensitivity analyses, we infer that the parameter settings kept constant during the simulation experiments were, in the main, sound and should not have influenced the results used to answer the research questions.

The main contribution of this research is that simulation experiments showed important associations between workplace social mixing and community-level incidence rates during influenza pandemics and that direct effects from reduced workplace virus transmission are accompanied by similarly large secondary community-wide reductions in transmissions. The results were generated in a controlled environment where factors not included in the simulation experiments were kept constant. We conclude that workplace cultures should be taken into consideration when modelling influenza pandemics and relevant differences in social mixing patterns between these cultures should be represented in models of virus transmission. More empirical research is warranted on social mixing at workplaces in association with influenza pandemics.

\section{SUPPLEMENTARY MATERIAL}

For supplementary material accompanying this paper visit http://dx.doi.org/10.1017/S0950268816000169.

\section{ACKNOWLEDGEMENTS}

This study was supported by grants from the Swedish Civil Contingencies Agency (T.T, grant no. 2010-2788); the Swedish Science Council (H.E., grant no. 2006-4433), (T.T, grant no. 2008-5252); and the Swedish Governmental Agency for Innovations (VINNOVA)
(T.T, grant no. 2011-03231). The funders had no role in study design, data collection and analysis, decision to publish, or preparation of the manuscript.

\section{DECLARATION OF INTEREST}

None.

\section{REFERENCES}

1. Tellier R. Review of aerosol transmission of influenza A virus. Emerging Infectious Diseases 2006; 12: 16571662.

2. Hall CB. The spread of influenza and other respiratory viruses: complexities and conjectures. Clinical Infectious Diseases 2007; 45: 353-359.

3. Schwartz EJ, Morgan M, Lapin S. Pandemic 2009 H1N1 influenza in two settings in a small community: the workplace and the university campus. Epidemiology and Infection 2015; 143: 1606-1609.

4. Strömgren M, et al. Place-based social contacts and mixing in Sweden. Department of Geography and Economic History, Umeå University, Sweden, 2015.

5. Sailer K, McCulloh I. Social networks and spatial configuration - how office layouts drive social interaction. Social Networks 2012; 34: 47-58.

6. Ibarra H. Homophily and differential returns: sex differences in network structure and access in an advertising firm. Administrative Science Quarterly 1992; 37: 422447.

7. McPherson M, Smith-Lovin L, Cook JM. Birds of a feather: homophily in social networks. Annual Review of Sociology 2001; 27: 415-444.

8. Danon L, et al. Social encounter networks: collective properties and disease transmission. Journal of the Royal Society Interface 2012; 9: 2826-2833.

9. Smieszek T, et al. Collecting close-contact social mixing data with contact diaries: reporting errors and biases. Epidemiology and Infection 2012; 140: 744-752.

10. Timpka T, et al. A neighborhood susceptibility index for planning of local physical interventions in response to pandemic influenza outbreaks. American Medical Informatics Association Annual Symposium Proceedings 2010; 2010: 792-796.

11. Timpka T, et al. Population-based simulations of influenza pandemics: validity and significance for public health policy. Bulletin of the World Health Organization 2009; 87: 305-311.

12. Eriksson H, et al. Assumptions management in simulation of infectious disease outbreaks. American Medical Informatics Association Annual Symposium Proceedings 2009; 2009: 173-177.

13. Eriksson H, et al. A cloud-based simulation architecture for pandemic influenza simulation. American Medical Informatics Association Annual Symposium Proceedings 2011; 2011: 364-373. 
14. Kucharski AJ, et al. The contribution of social behaviour to the transmission of influenza $\mathrm{A}$ in a human population. PLoS Pathogens 2014; 10: e1004206.

15. Kucharski AJ, Edmunds WJ. Cross-immunity and age patterns of influenza $\mathrm{A}(\mathrm{H} 5 \mathrm{~N} 1)$ infection. Epidemiology and Infection 2015; 143: 1119-1124.

16. Kumar S, et al. Is population structure sufficient to generate area-level inequalities in influenza rates? An examination using agent-based models. BMC Public Health 2015; 15: 947.

17. Eubank S, et al. Modelling disease outbreaks in realistic urban social networks. Nature 2004; 429: 180-184.

18. Balcan D. et al. Multiscale mobility networks and the spatial spreading of infectious diseases. Proceedings of the National Academy of Sciences USA 2009; 106: 21484-21489.

19. Grefenstette JJ, et al. FRED (a Framework for Reconstructing Epidemic Dynamics): an open-source software system for modeling infectious diseases and control strategies using census-based populations. BMC Public Health 2013; 13: 940.

20. Guo D, et al. Multi-scale modeling for the transmission of influenza and the evaluation of interventions toward it. Scientific Reports 2015; 5: 8980.

21. Elveback LR, et al. An influenza simulation model for immunization studies. American Journal of Epidemiology 1976; 103: 152-165.

22. Halloran ME, et al. Community interventions and the epidemic prevention potential. Vaccine 2002; 20: 3254-3262.

23. Hägerstrand T. Innovation Diffusion as a Spatial Process. Chicago: The University of Chicago Press, 1967.

24. Caillaud D, Craft ME, Meyers LA. Epidemiological effects of group size variation in social species. Journal of the Royal Society Interface 2013; 10: 20130206.

25. Holm E, Timpka T. A discrete time-space geography for epidemiology: from mixing groups to pockets of local order in pandemic simulations. Studies in Health Technology and Informatics 2007; 129: 464-468.

26. Halloran ME, et al. Modeling targeted layered containment of an influenza pandemic in the United States. Proceedings of the National Academy of Sciences USA 2008; 105: 4639-4644.

27. Read JM, Eames KT, Edmunds WJ. Dynamic social networks and the implications for the spread of infectious disease. Journal of the Royal Society Interface 2008; 5: 1001-1007.

28. Germann TC, et al. Mitigation strategies for pandemic influenza in the United States. Proceedings of the National Academy of Sciences USA 2006; 103: 59355940.

29. Carrat F, et al. Time lines of infection and disease in human influenza: a review of volunteer challenge studies. American Journal of Epidemiology 2008; 167: 775-785.
30. Timpka T, et al. Dealing with ecological fallacy in preparations for influenza pandemics: use of a flexible environment for adaptation of simulations to household structures in local contexts. Studies in Health Technology and Informatics 2007; 129: 218-222.

31. Eriksson H, et al. Ontology based modeling of pandemic simulation scenarios. Studies in Health Technology and Informatics 2007; 129: 755-759.

32. Kelso JK, Milne GJ, Kelly H. Simulation suggests that rapid activation of social distancing can arrest epidemic development due to a novel strain of influenza. BMC Public Health 2009; 9: 117.

33. Kelso JK, Halder N, Milne GJ. Vaccination strategies for future influenza pandemics: a severity-based cost effectiveness analysis. BMC Infectious Diseases 2013; 13: 81 .

34. Kumar S, et al. Policies to reduce influenza in the workplace: impact assessments using an agent-based model. American Journal of Public Health 2013; 103: 14061411.

35. Wallinga J, Teunis $\mathbf{P}$, Kretzschmar M. Using data on social contacts to estimate age-specific transmission parameters for respiratory-spread infectious agents. American Journal of Epidemiology 2006; 164: 936-944.

36. Mossong $\mathbf{J}$, et al. Social contacts and mixing patterns relevant to the spread of infectious diseases. PLoS Medicine 2008; 5: e74.

37. Kretzschmar M, Mikolajczyk RT. Contact profiles in eight European countries and implications for modelling the spread of airborne infectious diseases. PLoS ONE 2009; 4: e5931.

38. Milne GJ, Halder N, Kelso JK. The cost effectiveness of pandemic influenza interventions: a pandemic severity based analysis. PLoS ONE 2013; 8: e61504.

39. Jackson C, et al. The effects of school closures on influenza outbreaks and pandemics: systematic review of simulation studies. PLoS ONE 2014; 9: e97297.

40. Marathe A, et al. Impact of paid sick leave policy: a social planner's perspective. American Journal of Public Health 2014; 104: e1.

41. Kumar S, et al. The impact of workplace policies and other social factors on self-reported influenza-like illness incidence during the $2009 \mathrm{H} 1 \mathrm{~N} 1$ pandemic. American Journal of Public Health 2012; 102: 134-140.

42. Groenewold MR, et al. Exploring national surveillance for health-related workplace absenteeism: lessons learned from the 2009 influenza A pandemic. Disaster Medicine and Public Health Preparedness 2013; 7: 160-166.

43. Lau LL, et al. Household transmission of 2009 pandemic influenza A (H1N1): a systematic review and meta-analysis. Epidemiology 2012; 23: 531-542.

44. Wong VW, Cowling BJ, Aiello AE. Hand hygiene and risk of influenza virus infections in the community: a systematic review and meta-analysis. Epidemiology and Infection 2014; 142: 922-932. 